\title{
Cooperative Downlink Multicell Preprocessing Relying on Reduced-Rate Back-Haul Data Exchange
}

\author{
Rong Zhang, Member, IEEE, and Lajos Hanzo, Fellow, IEEE
}

\begin{abstract}
Different-complexity multicell preprocessing (MCP) schemes employing distributed signal-to-interference leakageplus-noise ratio (SILNR) precoding techniques are proposed, which require reduced back-haul data exchange in comparison with the conventional MCP structure. Our results demonstrate that the proposed structures are capable of increasing the throughput achievable in the cell-edge area while offering different geographic rate profile distributions, as well as meeting different delay requirements.
\end{abstract}

Index Terms-Cooperative communications, cross-layer design, limited backhaul, multicell multiple-input-multiple-output (MIMO), transmit preprocessing.

\section{INTRODUCTION}

$\mathbf{M}$ ULTIPLE-INPUT-MULTIPLE-OUTPUT (MIMO)system-based multicell preprocessing (MCP) [1], [2] constitutes a promising enabler for improving the throughput of cell-edge mobile stations (MSs) by jointly preprocessing the data of all the cooperating base stations (BSs) involved [3]. Moreover, MCP requires the channel state information (CSI) of all the links to all supported MSs at all the distributed transmitters to facilitate downlink (DL) joint precoding. This is typically achieved by a central unit (CU), which connects all the cooperating BSs considered via a limited-rate back haul [4], [5].

In the context of linear precoding, the so-called signalto-interference-leakage-plus-noise ratio (SILNR) maximization technique [6], which carefully balances the received signal power of the target MS against the interference power imposed on the remaining MSs, is capable of combining the benefits of both the egoistic beamforming (BF) technique and the altruistic zero-forcing technique [7], [8]. The SILNR maximization technique may be implemented in either a joint or distributed fashion. For the joint SILNR [9], the precoding matrix of all BSs cooperating for transmitting to a particular MS has to jointly be determined at the $\mathrm{CU}$ with the aid of all the MIMO channels involved. This is quite a demanding requirement, necessitating the back-haul exchange of both the data streams of all the MSs and the DL CSI of all the MSs. In contrast, for the distributed SILNR (DSILNR) [10], the BS's precoding matrix

Manuscript received March 16, 2010; revised September 21, 2010; accepted November 12, 2010. Date of publication November 18, 2010; date of current version February 18, 2011. This work was supported by the Engineering and Physical Sciences Research Council under the auspice of the U.K.-India Centre of Excellence in Wireless Communications. The review of this paper was coordinated by Dr. C.-C. Chong.

The authors are with the School of Electronics and Computer Science, University of Southampton, SO17 1BJ Southampton, U.K. (e-mail: rz@ecs. soton.ac.uk; lh@ecs.soton.ac.uk).

Color versions of one or more of the figures in this paper are available online at http://ieeexplore.ieee.org.

Digital Object Identifier 10.1109/TVT.2010.2093935 calculated for transmission to a particular MS may locally be determined at each individual BS, where only the channels spanning from the corresponding BS to the MSs are involved in the precoding matrix calculation. Hence, the DSILNR technique substantially reduces the signaling requirements from a CSI exchange perspective.

To the best of the authors' knowledge, the challenges of MCP relying on reduced data-rather than CSI-exchange have not been explored in the open literature. Hence, for the sake of reducing the burdens imposed on practical limited-rate backhaul design, in this paper, we do the following.

1) We propose a range of reduced-complexity MCP structures employing DSILNR relying on a reduced amount of data exchange, where the different BSs have to carry out different amounts of processing and information exchange.

2) We investigate the performance of various reducedcomplexity MCP structures in terms of their achievable outage rate and quantify the achievable throughput improvements over conventional single-cell preprocessing (SCP) schemes. Their geographic rate profile distributions are also characterized.

3) We also investigate the delay-limited performance of the best-supported and worst-supported MS for various reduced-complexity MCP structures, which are capable of supporting different quality of service (QoS) requirements.

We organize this paper as follows: In Section II, we provide a unified system model for SCP and MCP. Then, various MCP structures are proposed along with their precoding techniques employed. In Section III, we characterize the attainable performance of our MCP structures. Finally, we conclude in Section IV.

Notation: Throughout this paper, lower (upper) case boldface letters represent column vectors (matrices). The superscript $(\cdot)^{T}$ denotes transposition, and $(\cdot)^{H}$ represents the conjugate transpose. In addition, I represents the identity matrix. The unconventional abbreviations are listed in Table I.

\section{SYSTEM DESCRIPTION}

\section{A. Unified Model}

Let us first introduce the cellular topology in Fig. 1, where the hexagonal three-sector cellular model associated with a unity frequency reuse is employed. Let $\mathcal{B}_{c}$ denote the set hosting the BSs involved in the cooperative transmission. Practically, $\left|\mathcal{B}_{c}\right|=3$ denotes a realistic scenario, where $|\cdot|$ represents the cardinality of a set. These three adjacent BSs form a joint 
TABLE I

NOTATION SUMMARY

\begin{tabular}{|c|l|}
\hline ARQ & Automatic Repeat reQuest \\
\hline ASPDT & Average Successful Packet Delivery Time \\
\hline BPS & Bits Per Symbol \\
\hline BF/ZF & Beam-Forming/Zero-Forcing \\
\hline CSI & Channel State Information \\
\hline CU & Central Unit \\
\hline EL & Equally Loaded \\
\hline IL-I & Incrementally Loaded structure of type one \\
\hline IL-II & Incrementally Loaded structure of type two \\
\hline PSR & Packet Success Ratio \\
\hline MCP & Multi-Cell Pre-processing \\
\hline SCP & Single Cell Pre-processing \\
\hline SER & Symbol Error Ratio \\
\hline SILNR & Signal-to-Interference Leakage-plus-Noise-Ratio \\
\hline UEL-I & UnEqually Loaded structure of type one \\
\hline UEL-II & UnEqually Loaded structure of type two \\
\hline
\end{tabular}

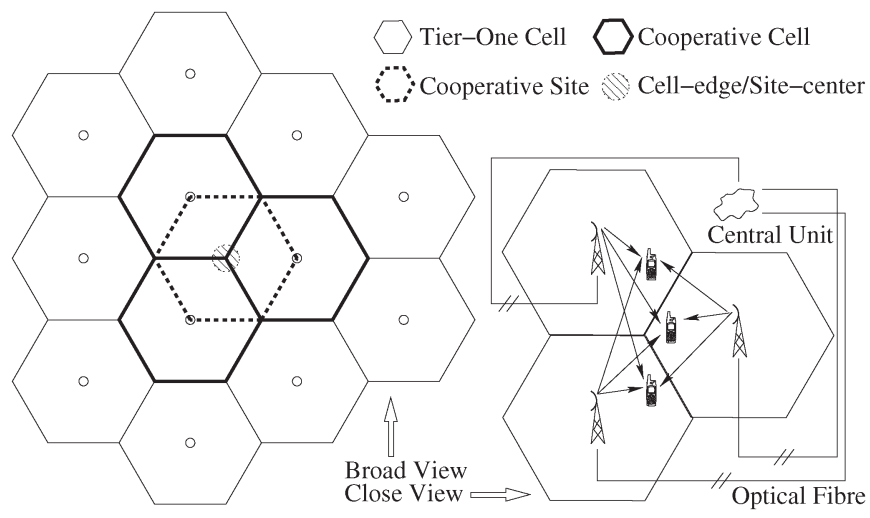

Fig. 1. Cellular topology considered and multicell processing.

cooperative transmission site, as indicated by the hexagonal area surrounded by the dashed line in Fig. 1. Hence, the cell edge of the conventional cells effectively becomes the cell center of the newly formed cooperative site, as indicated by the shaded circle in Fig. 1. These cooperative BSs are connected to a common $\mathrm{CU}$ via a limited-rate back haul.

Consider a general cooperative scenario constituted by $\left|\mathcal{B}_{c}\right|=N_{b}$ BSs, where each BS is equipped with $N_{t}$ transmit antennas. Let us assume that a total of $N_{u}=N_{b}$ MSs-each equipped with $N_{r}$ receive antennas-are involved in the cooperative scenario, where each of the $N_{u}$ MSs roams within the coverage area of a different BS, which is often referred to as its anchor BS, as portrayed in Fig. 1. Furthermore, we assume that each MS has to receive $N_{s}$ multiplexed DL data streams. Hence, the cooperative scenario may be described by the parameter combination $\left\{N_{b}, N_{t}, N_{u}, N_{r}, N_{s}\right\}$. Additionally, we let $N_{T}=\left(N_{b} \times N_{t}\right), N_{R}=\left(N_{u} \times N_{r}\right)$, and $N_{S}=\left(N_{u} \times N_{s}\right)$ denote the total number of BS transmitter antennas, MS receiver antennas, and MS data streams, respectively.

Hence, a unified discrete-time model for the signal received by $\mathrm{MS}_{j}$ for both SCP and MCP may be written as

$$
\mathbf{y}_{j}=\mathbf{H}_{E} \mathbf{G}_{E} \mathbf{x}+\mathbf{n}_{j}
$$

where $\mathbf{y}_{j} \in \mathbb{C}^{N_{r}}$ and $\mathbf{n}_{j} \in \mathbb{C}^{N_{r}}$ denote the received signal vector of $\mathrm{MS}_{j}$ and the circularly symmetric complex Gaussian noise vector having a covariance matrix of $\mathbf{R}_{n}=N_{0} \mathbf{I}$, respectively. Furthermore, $\mathbf{x}=\left[\mathbf{x}_{1}^{T}, \ldots, \mathbf{x}_{N_{u}}^{T}\right]^{T}$ hosts all the data streams of all MSs, where $\mathbf{x}_{j} \in \mathbb{C}^{N_{s}}, j \in\left[1, N_{u}\right]$, denotes the $N_{s}$ data streams destined for $\mathrm{MS}_{j}$ that have independent identically distributed (i.i.d.) zero-mean unit-variance complex Gaussian entries, which are assumed to be independent of both the noise and the channel. The entries of $\mathbf{x}$ obey $\mathbb{E}\left[\mathbf{x}_{i} \mathbf{x}_{j}^{H}\right]=$ I, where $\mathbb{E}[\cdot]$ stands for the expectation operation. Furthermore, $\mathbf{H}_{E} \in \mathbb{C}^{N_{r} \times N_{T}}$ represents the equivalent MIMO channel matrix, and similarly, $\mathbf{G}_{E} \in \mathbb{C}^{N_{T} \times N_{S}}$ is the equivalent linear precoding matrix.

More explicitly, we let $\mathbf{H}_{j, i} \in \mathbb{C}^{N_{r} \times N_{t}}, j \in\left[1, N_{u}\right], i \in$ $\mathcal{B}_{c}$, denote the MIMO channel between $\mathrm{MS}_{j}$ and $\mathrm{BS}_{i}$. We then let $\mathbf{G}_{i, j} \in \mathbb{C}^{N_{t} \times N_{s}}, i \in \mathcal{B}_{c}, j \in\left[1, N_{u}\right]$ denote the linear precoding matrix employed at $\mathrm{BS}_{i}$, which was calculated for transmission to $\mathrm{MS}_{j}$. Hence, the global MIMO channel matrix $\mathbf{H} \in \mathbb{C}^{N_{R} \times N_{T}}$ and the global linear precoding matrix $\mathbf{G} \in$ $\mathbb{C}^{N_{T} \times N_{S}}$ may be expressed as

$$
\begin{aligned}
\mathbf{H} & =\left[\begin{array}{ccc}
\mathbf{H}_{1,1} & \cdots & \mathbf{H}_{1, N_{b}} \\
\vdots & \vdots & \vdots \\
\mathbf{H}_{N_{u}, 1} & \cdots & \mathbf{H}_{N_{u}, N_{b}}
\end{array}\right] \\
\mathbf{G} & =\left[\begin{array}{ccc}
\mathbf{G}_{1,1} & \cdots & \mathbf{G}_{1, N_{u}} \\
\vdots & \vdots & \vdots \\
\mathbf{G}_{N_{b}, 1} & \cdots & \mathbf{G}_{N_{b}, N_{u}}
\end{array}\right] .
\end{aligned}
$$

The equivalent MIMO channel matrix $\mathbf{H}_{E}$ of (1) may then be expressed as $\mathbf{H}_{E}=\mathbf{H}_{j, \forall}$, where $\mathbf{H}_{j, \forall}$ denotes the $j$ th row of $\mathbf{H}$. According to the equivalent linear precoding matrix $\mathbf{G}_{E}$ of (1), for SCP, we may write $\mathbf{G}_{E}=\operatorname{diag}[\mathbf{G}]$, which is a diagonal matrix hosting the diagonal entries of matrix $\mathbf{G}$. By contrast, for $\mathrm{MCP}$, we have $\mathbf{G}_{E}=\mathbf{G}$, which subsumes the various $\mathrm{MCP}$ structures to be introduced in the next section. Hence, the discrete-time signal received at $\mathrm{MS}_{j}$ for $\mathrm{SCP}$ and $\mathrm{MCP}$ may, respectively, be written as

$$
\begin{gathered}
\mathbf{y}_{j}^{s}=\mathbf{H}_{j, j} \mathbf{G}_{j, j} \mathbf{x}_{j}+\sum_{i \in \mathcal{B}_{c,-j}} \mathbf{H}_{j, i} \mathbf{G}_{i, i} \mathbf{x}_{i}+\mathbf{n}_{j} \\
\mathbf{y}_{j}^{m}=\mathbf{H}_{j, \forall} \mathbf{G}_{\forall, j} \mathbf{x}_{j}+\sum_{k=1, k \neq j}^{N_{u}} \mathbf{H}_{j, \forall} \mathbf{G}_{\forall, k} \mathbf{x}_{k}+\mathbf{n}_{j}
\end{gathered}
$$

where $\mathbf{G}_{\forall, j}$ denotes the $j$ th column of matrix $\mathbf{G}$, and $\mathcal{B}_{c,-j}$ hosts the BSs within the cooperative site, excluding $\mathrm{BS}_{j}$.

\section{B. Conventional MCP Structure and DSILNR}

In the conventional MCP, each BS is responsible for the coprocessing of all MSs' data streams $\mathbf{x}$; in other words, each MS benefits from the joint transmission from all BSs. Hence, this structure may be referred to as equally loaded (EL) cooperative BS structure. In the EL MCP structure, each BS has to know all the MSs' data streams, which requires a substantial amount of data exchange before their precoded DL transmission ensues. Hence, the corresponding global linear precoding matrix $\mathbf{G}$ only has nonzero entries $\mathbf{G}_{i, j}, i \in \mathcal{B}_{c}$, $j \in\left[1, N_{u}\right]$, as demonstrated on the left side of Fig. 2 . 


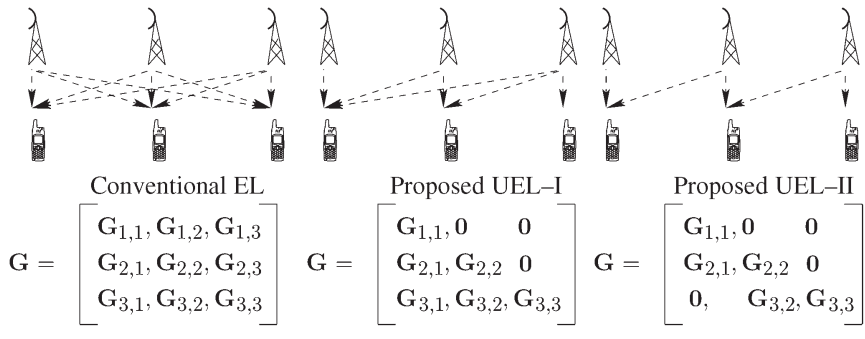

Fig. 2. Conventional and reduced-complexity MCP structure as well as their global linear precoding matrix $\mathbf{G}$.

The linear SILNR maximization precoding technique [6] employed in this conventional structure aims at maximizing the signal power received at the intended MS and at the same time for minimizing the interference imposed on all the other MSs. In the context of DSILNR [10], this maximization takes place locally at each individual BS. More explicitly, the SILNR $\gamma$ at $\mathrm{MS}_{j}$ is given by

$$
\gamma=\frac{\operatorname{Tr}\left[\mathbf{G}_{i, j}^{H} \mathbf{H}_{j, i}^{H} \mathbf{H}_{j, i} \mathbf{G}_{i, j}\right]}{\operatorname{Tr}\left[\mathbf{G}_{i, j}^{H}\left(N_{0} N_{r} \mathbf{I} / P_{i, j}+\sum_{k=\chi_{l}, k \neq j}^{\chi_{u}} \mathbf{H}_{k, i}^{H} \mathbf{H}_{k, i}\right) \mathbf{G}_{i, j}\right]}
$$

where the numerator denotes the signal power received at $\mathrm{MS}_{j}$ benefiting from the transmission at $\mathrm{BS}_{i}$, whereas the denominator represents the interference leakage power imposed on all the other MSs due to the transmission of $\mathrm{BS}_{i}$ to $\mathrm{MS}_{j}$ with $\left[\chi_{u}, \chi_{l}\right]=\left[N_{u}, 1\right]$. Hence, the optimization problem may be stated as

$$
\mathbf{G}_{i, j}=\arg \max _{\mathbf{G}_{i, j}} \gamma
$$

It can be seen that the maximization problem of (5) requires the knowledge of the power allocation $P_{i, j}$, namely, the power allocated for the transmission of $\mathrm{BS}_{i}$ to $\mathrm{MS}_{j}$. To satisfy the perBS power constraint $P_{B S}$, which is assumed to be the same for all BSs, we adopt the power-allocation strategy of [10]

$$
P_{i, j}=\frac{\operatorname{Tr}\left[\mathbf{H}_{j, i} \mathbf{H}_{j, i}^{H}\right]}{\operatorname{Tr}\left[\sum_{k=\chi_{l}}^{\chi_{u}} \mathbf{H}_{k, i} \mathbf{H}_{k, i}^{H}\right]} P_{B S}
$$

where the numerator denotes the channel gain associated with the transmission of $\mathrm{BS}_{i}$ to $\mathrm{MS}_{j}$, whereas the denominator represents the aggregate channel gain of the transmission of $\mathrm{BS}_{i}$ to all MSs. Again, we have $\chi_{u}=N_{u}$ and $\chi_{l}=1$ for the conventional MCP structure. Once the power allocation was determined, the optimization problem of (5) may further be decoupled into individual optimization steps by constraining $\mathbf{G}_{i, j}$ to be an orthonormal matrix, as discussed in [6]. Hence, we may obtain the optimization solution as $\mathbf{G}_{i, j}=$ $\operatorname{eigv}\left(\mathbf{B}^{-1} \mathbf{A}\right)$, representing the eigenvectors corresponding to the $N_{s}$ largest eigenvalues of $\mathbf{B}^{-1} \mathbf{A}$, with $\mathbf{B}=N_{0} N_{r} \mathbf{I} / P_{i, j}+$ $\sum_{k=1, k \neq j}^{N_{u}} \mathbf{H}_{k, i}^{H} \mathbf{H}_{k, i}$ and $\mathbf{A}=\mathbf{H}_{j, i}^{H} \mathbf{H}_{j, i}$. Finally, the resultant linear precoding matrix $\mathbf{G}_{i, j}$ is multiplied by the appropriately allocated power of $P_{i, j} / N_{s}$, where we have implicitly assumed that the power $P_{i, j}$ is equally distributed among the $N_{s}$ DL data streams to be transmitted to $\mathrm{MS}_{j}$.

\section{Reduced-Complexity MCP Structures}

Apart from the rather demanding EL MCP structure, which requires the exchange of all the data streams $\mathrm{x}$ of all MSs, we propose various reduced-complexity structures, where each BS is responsible for the DL transmission of a different number of MSs' data streams.

1) DSILNR-UEL-I: In the first reduced-complexity structure considered, $\mathrm{BS}_{i}$ is only responsible for transmission to MSs within its own cell as well as for transmissions to all the MSs roaming within the previous $(i-1)$ BSs of the BS set, where the data streams $\left[\mathbf{x}_{1}, \ldots, \mathbf{x}_{i-1}\right]$ of all the $(i-1)$ MSs have to be known at $\mathrm{BS}_{i}$. Hence, this may be referred to as the unequally loaded structure of type one (UEL-I). For example, when considering a cooperative BS set of size $\left|\mathcal{B}_{c}\right|=3$, as seen in the middle of Fig. 2, $\mathrm{BS}_{i=1}$ is only responsible for transmissions to its own cell edge $\mathrm{MS}_{j=1}$, whereas $\mathrm{BS}_{i=2}$ is responsible for transmissions to its own cell edge $\mathrm{MS}_{j=2}$ and to $\mathrm{MS}_{j=1}$, and finally, $\mathrm{BS}_{i=3}$ jointly transmits to all $\mathrm{MS}_{j=1,2,3}$. In this structure, the $N_{s}$ data streams $\mathbf{x}_{1}$ of $\mathrm{MS}_{j=1}$ have to be available at $\mathrm{BS}_{i=2}$, and the data streams $\left[\mathrm{x}_{1}, \mathbf{x}_{2}\right]$ of $\mathrm{MS}_{j=1}$ and $\mathrm{MS}_{j=2}$ have to be available at $\mathrm{BS}_{i=3}$. Hence, the global linear precoding matrix $\mathbf{G}$ becomes a lower triangular matrix, as seen in the middle of Fig. 2. The DSILNR precoding technique employed remains similar to that discussed in Section II-B, with the slight change that the parameters $\chi_{u}$ and $\chi_{l}$ of (6) become $\chi_{u}=i$ and $\chi_{l}=1$.

2) DSILNR-UEL-II: In the second reduced-complexity structure advocated, $\mathrm{BS}_{i}$ is only responsible for transmissions to MSs in its own cell as well as for transmissions to MSs belonging to $\mathrm{BS}_{i-1}$, where only the data streams $\mathbf{x}_{i-1}$ of MS belonging to $\mathrm{BS}_{i-1}$ have to be available at $\mathrm{BS}_{i}$. This regime may be referred to as the unequally loaded structure of type two (UEL-II). For example, when considering the cooperative BS set of size $\left|\mathcal{B}_{c}\right|=3$ seen in Fig. $2, \mathrm{BS}_{i=1}$ and $\mathrm{BS}_{i=2}$ have the same configuration as that of UEL-I, whereas $\mathrm{BS}_{i=3}$ is only responsible for transmissions to $\mathrm{MS}_{j=2}$ and $\mathrm{MS}_{j=3}$, where only the data streams $\mathbf{x}_{2}$ of $\mathrm{MS}_{j=2}$ have to be available at $\mathrm{BS}_{i=3}$. Hence, the global linear precoding matrix $\mathbf{G}$ becomes a zigzag-shaped matrix, as seen in the right of Fig. 2. The DSILNR precoding technique essentially remains the same as discussed in Section II-B, where the parameters $\chi_{u}$ and $\chi_{l}$ of (6) become $\chi_{u}=i$ and $\chi_{l}=\max (i-1,1)$.

3) DSILNR-IL-I and IL-II: The third reduced-complexity structure is similar to the UEL-I structure, with the slight difference that the MCP is operated progressively, where the participation of $\mathrm{BS}_{i}$ does not affect the transmission precoding matrix employed at the BSs that already have ongoing transmissions. For example, when considering the cooperative BS set of size $\left|\mathcal{B}_{c}\right|=3$, where $\mathrm{BS}_{i=3}$ participates in the MCP, then the precoding matrices already employed at $\mathrm{BS}_{i=1}$ and $\mathrm{BS}_{i=2}$ remain unchanged. Hence, this structure may be referred to as the incrementally loaded structure of type one (IL-I). Note that this structure has the same data stream exchange requirement as that of the UEL-I structure while imposing 
only marginal modifications on the network. Likewise, we may define the incrementally loaded structure of type two (IL-II) as the progressive version of the UEL-II structure. As for the other schemes, the DSILNR precoding technique remains the same as that discussed in Section II-B, with the minor change that the parameters $\chi_{u}$ and $\chi_{l}$ of both (6) and (4) become $\chi_{u}=i$ and $\chi_{l}=1$ for the IL-I structure and $\chi_{u}=i$ and $\chi_{l}=$ $\max (i-1,1)$ for the IL-II structure, respectively.

\section{Performance Evaluation}

In this section, we characterize the various MCP structures employing the DSILNR precoding technique in both the fully loaded scenario $\left(N_{T}=N_{R}\right)$ of $\left\{N_{b}, N_{t}, N_{u}, N_{r}, N_{s}\right\}=$ $\{3,2,3,2,1\}$ and the lightly loaded scenario $\left(N_{T}>N_{R}\right)$ of $\left\{N_{b}, N_{t}, N_{u}, N_{r}, N_{s}\right\}=\{3,4,3,2,1\}$.

We considered the so-called Urban Micro setup [11], where the BS-to-BS distance was defined as $D=1000 \mathrm{~m}$. The MIMO channel of each BS-MS pair is constituted by three components, i.e., by $\mathbf{H}_{j, i}=\left(A_{j, i}^{l} A_{j, i}^{s}\right)^{1 / 2} \mathbf{H}_{j, i}^{f}$, where $\mathbf{H}_{j, i}^{f} \in \mathbb{C}^{N_{r} \times N_{t}}$ represents the fast fading component, which is assumed to be frequency flat with zero-mean and unity-variance complex Gaussian entries, and $A_{j, i}^{s}=10^{\xi / 10}$ is the lognormal shadow fading component, where $\xi$ is generated by a zero-mean realvalued Gaussian random variable having a standard derivation of $\sigma_{s}=8 \mathrm{~dB}$. The path loss model is given by $A_{j, i}^{l}=\beta d_{j, i}^{\alpha}$, where $d$ denotes the BS-MS distance in meters, and $[\alpha, \beta]=$ $\left[-3,1.35 \times 10^{7}\right][12]$. Furthermore, we let the signal-to-noise ratio at the cell-edge area be $25 \mathrm{~dB}$.

In each simulation, $N_{u}=3$ MS locations are generated randomly and independently, where each of the MSs is random uniformly positioned in the area determined by the radius range of $r \in(0,0.1 D]$ and angle range of $120^{\circ}$, corresponding to the sector covered by its anchor BS. Furthermore, we investigate a total of 100 independent simulations, where each simulation was terminated after 5000 simulation runs.

\section{A. Achievable Outage Rate}

1) Performance Metric: To arrive at insightful results, we plot the "outage rate," which is defined as the cumulative distribution function (CDF) of the ergodic achievable rate. We consider equal power allocation in the SCP scenario, namely, $\mathbf{G}_{i, i}=\sqrt{P_{B S} / N_{t}} \mathbf{I}$, when a single-user detector is employed as a benchmarker, which is unaware of the co-channel interference. The achievable rate of $\mathrm{MS}_{j}$ of the SCP scheme is given as

$$
\mathcal{R}_{j}^{s}=\log \left|\mathbf{I}+\frac{\mathbf{H}_{j, j} \mathbf{G}_{j, j} \mathbf{G}_{j, j}^{H} \mathbf{H}_{j, j}^{H}}{N_{0} \mathbf{I}+\sum_{i \in \mathcal{B}_{c,-j}} \mathbf{H}_{j, i} \mathbf{G}_{i, i} \mathbf{G}_{i, i}^{H} \mathbf{H}_{j, i}^{H}}\right| .
$$

On the other hand, in the MCP scenario, the achievable rate of $\mathrm{MS}_{j}$ is given as

$$
\mathcal{R}_{j}^{m}=\log \left|\mathbf{I}+\frac{\mathbf{H}_{j, \forall} \mathbf{G}_{\forall, j} \mathbf{G}_{\forall, j}^{H} \mathbf{H}_{j, \forall}^{H}}{N_{0} \mathbf{I}+\sum_{k=1, k \neq j}^{N_{u}} \mathbf{H}_{j, \forall} \mathbf{G}_{\forall, k} \mathbf{G}_{\forall, k}^{H} \mathbf{H}_{j, \forall}^{H}}\right| .
$$

2) Performance Overview: Fig. 3 shows the outage rate of each of the $N_{u}=3 \mathrm{MSs}$ (top, middle, bottom) for both conventional and proposed structures in the fully loaded scenario (left) and the lightly loaded scenario (right), respectively. In all the figures, the crosses and the asterisks in the legend stand for the conventional EL MCP arrangement and the benchmarker SCP scenario, respectively. It can be seen in Fig. 3 for both fully loaded and lightly loaded scenarios that all three MSs supported by both conventional and proposed structures achieve a higher outage rate than that of the SCP arrangement marked by asterisks. In general, $\mathrm{MS}_{j=2}$ characterized in the middle subplot of both Fig. 3(a) and (b) exhibits a similar outage rate for all structures considered, whereas $\mathrm{MS}_{j=1}$ and $\mathrm{MS}_{j=3}$ exhibit quite a different outage rate behavior for the various structures, as subsequently detailed.

3) Performance of $M S_{j=1}$ : As seen in the top subplot of both Fig. 3(a) and (b) for $\mathrm{MS}_{j=1}$, the type-one reducedcomplexity MCP structures are indicated by the solid line for UEL-I and the dash-dot line for IL-I, both of which achieve a noticeable outage rate improvement over the conventional EL MCP structure indicated by the crosses. However, the type-two reduced-complexity MCP structures indicated by the dashed line for UEL-II and the dotted line for IL-II only achieve marginal or no outage rate improvement when compared with the conventional EL MCP structure.

Remarks: The outage rate improvement of $\mathrm{MS}_{j=1}$ in the proposed reduced-complexity MCP structures is a consequence of the higher total transmission power $P_{i, 1}$ radiated from $\mathrm{BS}_{i=1}$ and $\mathrm{BS}_{i=2}$ for their transmission to $\mathrm{MS}_{j=1}$ compared with the conventional EL structure. For example, the full transmit power of $\mathrm{BS}_{i=1}$ is exclusively used for transmission to $\mathrm{MS}_{j=1}$, whereas the available power has to be shared for transmission to all three MSs in the conventional EL MCP structure.

4) Performance of $M S_{j=2}$ : Observe in the bottom subplot of both Fig. 3(a) and (b) for $\mathrm{MS}_{j=3}$ that all reduced-complexity MCP structures suffer from an outage rate loss when compared with the conventional EL structure. This is as expected, since, in contrast to the conventional structure, where $\mathrm{MS}_{j=3}$ benefits from multiple BSs' transmission, only the anchor BS, namely, $\mathrm{BS}_{i=3}$ associated with $\mathrm{MS}_{j=3}$, is responsible for transmission to $\mathrm{MS}_{j=3}$ in the proposed reduced-complexity MCP structures, and only a fraction of the total transmission power $P_{B S}$ is available for $\mathrm{BS}_{i=3}$ for transmission to $\mathrm{MS}_{j=3}$.

Remarks: Importantly, the achievable outage rate remains still higher than that of the SCP, where the full transmit power is available at $\mathrm{BS}_{i=3}$ for transmissions to $\mathrm{MS}_{j=3}$. This is because although the transmit power available at $\mathrm{BS}_{i=3}$ for transmission to $\mathrm{MS}_{j=3}$ is reduced in MCP in comparison with the SCP scenario, the interference imposed by the other BSs on $\mathrm{MS}_{j=3}$ is reduced more substantially as a benefit of the DSILNR precoding technique employed at all BSs. This also explains the fact that the UEL-I and UEL-II structures achieve a higher outage rate than the IL-I and IL-II structures since the precoding techniques employed in the latter two approaches do not take into account any of the newly activated cooperative BSs, which in turn do not facilitate the control of the interference imposed on those MSs, which have just initiated a call, although the ongoing transmissions are not affected at all. 


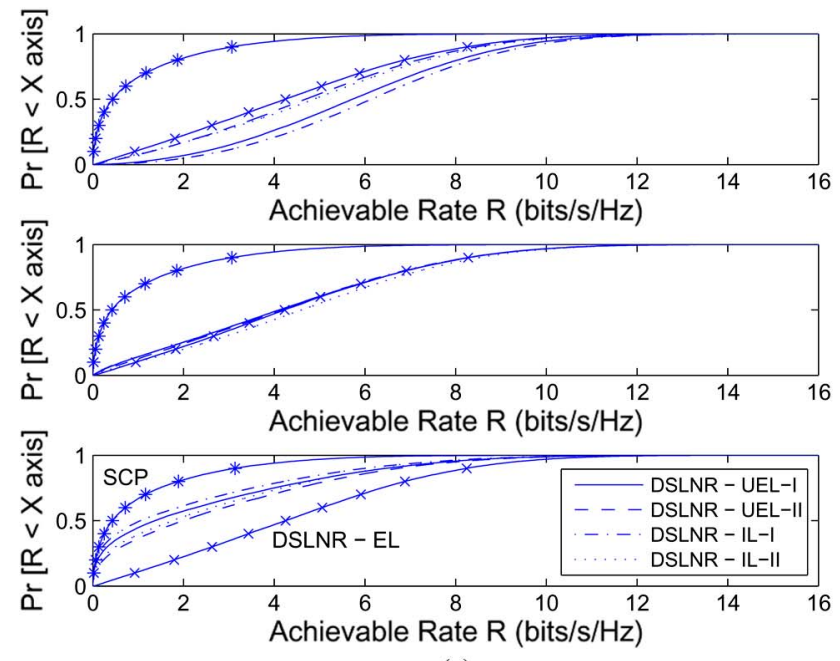

(a)

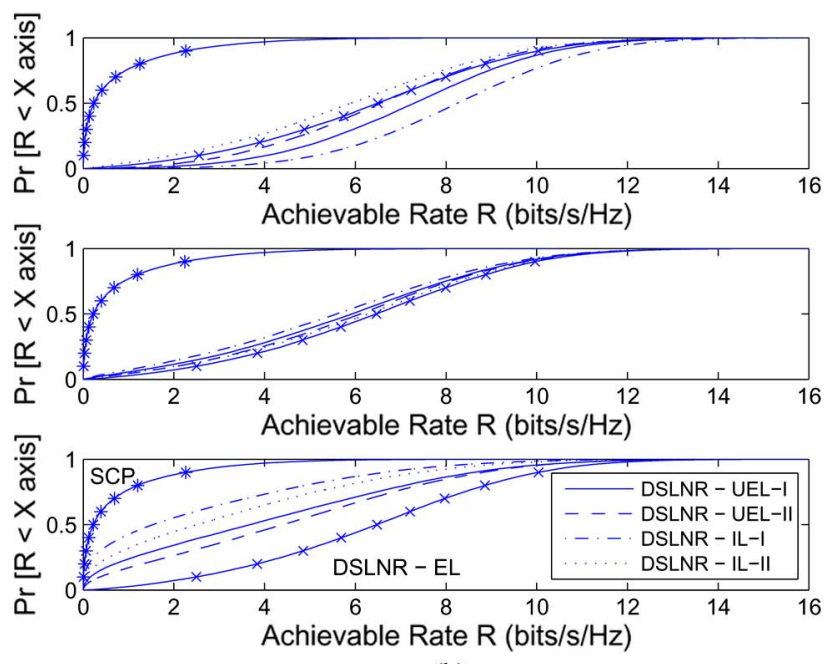

(b)

Fig. 3. Outage rate of each of the $N_{u}=3$ MSs (top, middle, bottom) for both the conventional and the proposed reduced-complexity structures. (a) Fully loaded scenario. (b) Lightly loaded scenario.

\section{B. Delay Limited Performance}

1) Performance Metric: We now further investigate the delay-limited performance for both $\mathrm{MS}_{j=1}$ and $\mathrm{MS}_{j=3}$. Given a system bandwidth $B$, a packet's transmission may be modeled by a queue and a wireless link using the M/G/1 model of [13], which has a Poissonian source packet arrival process having an arrival rate of $\lambda$, a general i.i.d. packet delivery time $T$, and a single server. Let us denote the maximum of the average delay tolerated by a particular service as $D$. Naturally, the average delay imposed by the system, which depends on both the packet delivery time $T$ and the queueing delay $D_{q}$, has to be lower than $D$. In fact, in practice, even the peak delay should be limited, although we do not consider applications that have hard delay requirements in this paper. To simplify our system model, we ignore the propagation delay, the data exchange delay of the cooperating BSs, and the packet acknowledgement feedback delay. Then, we have $T+D_{q} \leq D$. For the M/G/1 queue, the average delay constraint may be quantified by [13]

$$
\mathbb{E}[T]+\lambda\left[\mathbb{E}[T]^{2}+\sigma_{T}^{2}\right] /[2-2 \lambda \mathbb{E}[T]] \leq D
$$

where $\mathbb{E}[T]$ and $\sigma_{T}^{2}$ denote the average successful packet delivery time (ASPDT) and its variance, respectively. We assume encountering i.i.d. packet error events, and in contrast to our previous outage rate studies, we assume that the corrupted packets are retransmitted by an Automatic Repeat reQuest scheme, which keeps retransmitting a given packet until its successful reception is declared. Hence, the probability of the $l$ th transmission (i.e., the $(l-1)$ st retransmission) of a packet succeeding is given by $\operatorname{Pr}(l)=f(\gamma)[1-f(\gamma)]^{l-1}$, where $f(\gamma)$ is defined as the packet success ratio (PSR), which is a monotonically increasing function of the signal-to-interferenceplus-noise ratio (SINR) $\gamma$. In this paper, we consider Graymapped square $2^{b}$-QAM packets having a length of $N$ bits, where $b$ represents the number of bits per symbol (BPS). Hence, the PSR is given by $f(\gamma)=\left[1-p_{P A M}(\gamma)\right]^{2 N / b}$, where $p_{P A M}(\gamma)$ is the symbol error ratio (SER) of a $2^{b-1}$-ary pulse amplitude modulation (PAM) scheme [11]. The SINR $\gamma$ can be recorded in the same way as in the simulations conducted in Section III-A for quantifying the achievable outage rate for both conventional and proposed reduced-complexity MCP structures, since the calculation of SINR is an intermediate step toward the calculation of outage rate, but instead of calculating the CDF, we require the average SINR value. As a result, the ASPDT and its variance are given by [14]

$$
\begin{aligned}
\mathbb{E}[T] & =\tau / f(\gamma) \\
\sigma_{T}^{2} & =[1-f(\gamma)] \tau^{2} / f(\gamma)^{2}
\end{aligned}
$$

where the packet duration is $\tau=N / b R$, and $R$ represents the symbol rate. By substituting (10) and (11) into (9), it may readily be shown that $D$, namely, the maximum of the average delay tolerated by the specific service considered, has to obey the following constraint:

$$
N / b R D+\lambda N / b R-\lambda N^{2} / 2 b^{2} R^{2} D \leq f(\gamma)
$$

Given $D, b, N$, and $\lambda$, we may find a set of combined solutions $[b, R]$ for (12) [15] when the equality holds. To further refine the resultant solution set, additional constraints should be imposed. Naturally, the average delay is higher than the packet duration; hence, we have $D>\tau$. Second, the packet arrival rate required to support the specific service considered must not be higher than the packet service rate $\mu=1 / E[T]$ delivered by the system to avoid buffer overflow; hence, we have $\lambda<\mu$. Finally, let us define the normalized transmission rate as $R_{n}=R / B$, the normalized delay as $D_{n}=D B$, and the normalized service arrival rate as $\lambda_{n}=\lambda / B$.

Our goal is to find the minimum normalized transmission rate $R_{n}$ so that a given service maximum tolerable normalized delay $D_{n}$ is not exceeded, which is characterized in Fig. 4 for both fully and lightly loaded scenarios in both conventional and proposed structures when the normalized service arrival rate is set to $\lambda_{n}=0.005$, and the packet length is set to $N=240$ bits. 


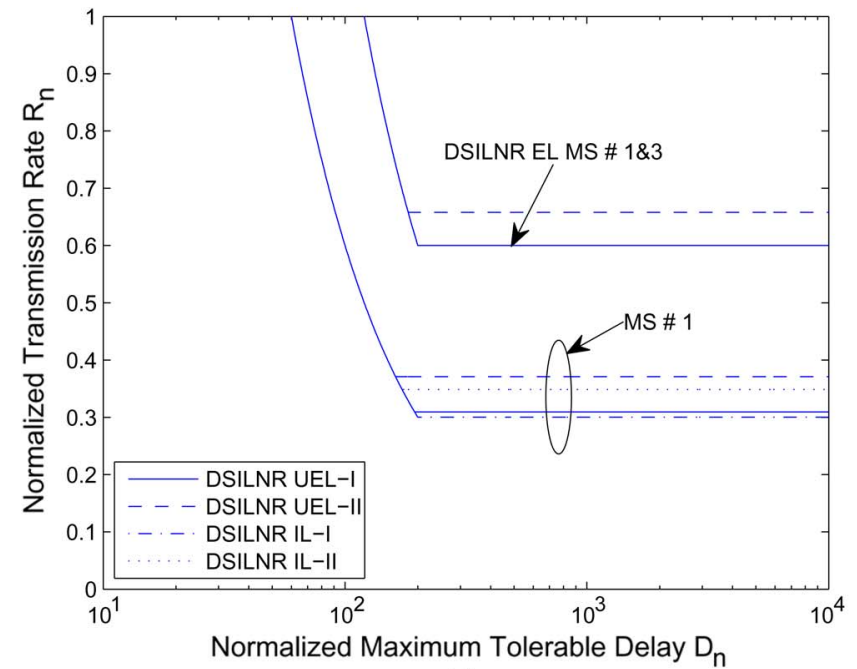

(a)

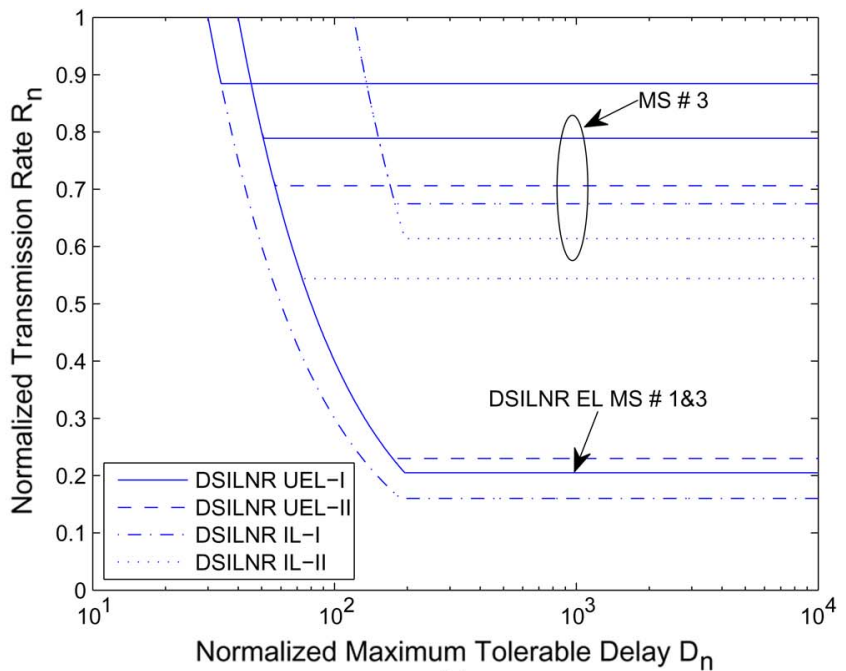

(b)

Fig. 4. Minimum required normalized transmission rate $R_{n}$ as a function of the normalized maximum tolerable delay $D_{n}$ for both $\mathrm{MS}_{j=1}$ and $\mathrm{MS}_{j=3}$ under both the conventional and the proposed structures. (a) Fully loaded scenario. (b) Lightly loaded scenario.

2) Delay Investigation: In the fully loaded scenario characterized in Fig. 4(a), we observe for $\mathrm{MS}_{j=1}$ that the proposed structures require a consistently lower normalized transmission rate $R_{n}$ to maintain a given maximum delay $D_{n}$ than the conventional EL structure. When the normalized transmission rate $R_{n}$ approaches its maximum value of unity, the system became capable of operating at a lower delay; hence, the proposed structures support a tighter delay constraint. On the other hand, for $\mathrm{MS}_{j=3}$, only the UEL-II structure is capable of imposing as low a delay as the conventional structure, and this is at the cost of requiring a higher normalized transmission rate $R_{n}$.

On the other hand, when considering the lightly loaded scenario of Fig. 4(b) and $\mathrm{MS}_{j=1}$, both of the reduced-complexity type-one structures operate at a lower delay than the conventional EL structure, as indicated by the solid line for UEL-I and by the dash-dot line for IL-I. By contrast, both reducedcomplexity type-two structures indicated by the dashed line for UEL-II and the dotted line for IL-II achieved a similar delay at the cost of consistently requiring a higher normalized transmission rate $R_{n}$. On the other hand, we observe for $\mathrm{MS}_{j=3}$ that although the outage rate performance of the proposed structures is inferior in comparison with that of the conventional MCP structure, as seen in the bottom subplot of Fig. 3(b), the delay imposed by both UEL-I and UEL-II structures is the same as that of the conventional EL structure, which is achieved at the cost of requiring an increased normalized transmission rate $R_{n}$.

\section{CONCLUSION}

Reduced-complexity MCP structures associated with reduced back-haul data exchange requirements and employing the DSILNR precoding technique have been proposed in the context of a unified system model. The achievable outage rate and the maximum system delay were characterized. Our results demonstrated that the proposed structure achieves a higher outage rate than the SCP benchmarker for all the MSs supported. The different MSs belonging to different cells benefit from different grades of MCP enhancements in terms of both the outage rate and the delay imposed. Naturally, the proposed reduced-complexity MCP structures cannot be expected to outperform the conventional MCP structure in all scenarios, but they tend to offer a different geographical rate distribution and different delays while requiring reduced data exchange. Hence, the proposed structures are capable of supporting different QoS requirements.

\section{REFERENCES}

[1] H. Zhang and H. Dai, "Cochannel interference mitigation and cooperative processing in downlink multicell multiuser MIMO networks," EURASIP J. Wireless Commun. Netw., vol. 2004, no. 2, pp. 222-235, Dec. 2004.

[2] W. Choi and J. Andrews, "Downlink performance and capacity of distributed antenna systems in a multicell environment," IEEE Trans. Wireless Commun., vol. 6, no. 1, pp. 69-73, Jan. 2007.

[3] S. Shamai and B. Zaidel, "Enhancing the cellular downlink capacity via co-processing at the transmitting end," in Proc. IEEE Veh. Technol. Conf.-Spring, Rhodes, Greece, Jun. 2001, vol. 3, pp. 1745-1749.

[4] M. Karakayali, G. Foschini, and R. Valenzuela, "Network coordination for spectrally efficient communications in cellular systems," IEEE Wireless Commun., vol. 13, no. 4, pp. 56-61, Aug. 2006.

[5] S. Parkvall, E. Dahlman, A. Furuskar, Y. Jading, M. Olsson, S. Wanstedt, and K. Zangi, "LTE-Advanced-Evolving LTE towards IMT-Advanced," in Proc. IEEE Veh. Technol. Conf._Fall, Calgary, AB, Canada, Sep. 2008, vol. 3, pp. 1-5.

[6] M. Sadek, A. Tarighat, and A. Sayed, "A leakage-based precoding scheme for downlink multi-user MIMO channels," IEEE Trans. Wireless Commun., vol. 6, no. 5, pp. 1711-1721, May 2007.

[7] E. Larsson and E. Jorswieck, "Competition versus cooperation on the MISO interference channel," IEEE J. Sel. Areas Commun., vol. 26, no. 7, pp. 1059-1069, Sep. 2008.

[8] Z. K. M. Ho and D. Gesbert, "Balancing egoism and altruism on the interference channel: The MIMO case," in Proc. IEEE Int. Conf. Commun., Cape Town, S. Africa, May 2010, pp. 1-5.

[9] H. Zhang, N. B. Mehta, A. F. Molisch, J. Zhang, and H. Dai, "Asynchronous interference mitigation in cooperative base station systems," IEEE Trans. Wireless Commun., vol. 7, no. 1, pp. 155-165, Jan. 2008.

[10] E. Bjornson, R. Zakhour, D. Gesbert, and B. Ottersten, "Cooperative multicell precoding: Rate region characterization and distributed strategies with instantaneous and statistical CSI," IEEE Trans. Signal Process., vol. 58, no. 8, pp. 4298-4310, Aug. 2010.

[11] R. Steele and L. Hanzo, Mobile Radio Communications. New York: Wiley, 1999.

[12] T. S. Rappaport, Wireless Communications: Principles and Practice. Englewood Cliffs, NJ: Prentice-Hall, 1996. 
[13] W. Stevens, TCP/IP Illustrated, Volume I: The Protocols. Reading, MA: Addison-Wesley, 1994.

[14] F. Meshkati, A. J. Goldsmith, H. V. Poor, and S. C. Schwartz, "A gametheoretic approach to energy-efficient modulation in CDMA networks with delay QoS constraints," IEEE J. Sel. Areas Commun., vol. 25, no. 6, pp. 1069-1078, Aug. 2007.

[15] R. Zhang and L. Hanzo, "Superposition aided delay-constrained hybrid automatic repeat request," IEEE Trans. Veh. Technol., vol. 59, no. 4, pp. 2109-2115, May 2010.

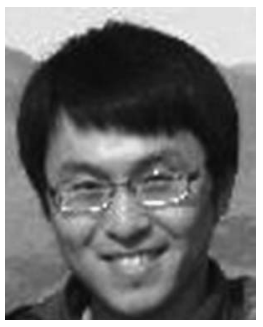

Rong Zhang (M'10) received the B.Eng. degree in communications engineering from Southeast University, Nanjing, China, in 2003 and the M.Sc. degree with distinction in radio frequency communications engineering and the Ph.D. degree in wireless communications from the University of Southampton, Southampton, U.K., in 2005 and 2009, respectively.

$\mathrm{He}$ was a System Engineer with the Mobile Communications Division, China Telecom, and is currently a Research Fellow with the Communications Research Group, School of Electronics and Computer Science, University of Southampton. His research interests include cellular network optimization, multiuser communications, multiantenna communications, and cooperative communications.

Dr. Zhang was the recipient of a joint Engineering and Physical Sciences Research Council and Mobile VCE scholarship in 2006. He is a member of the Institution of Engineering and Technology.

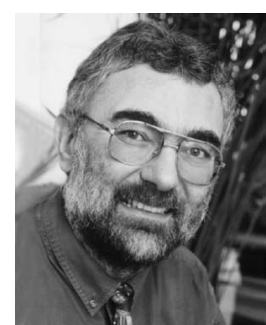

Lajos Hanzo (F'08) received the M.S. degree in electronics and the Ph.D. degree in 1976 and 1983, respectively, both from the Technical University of Budapest, Budapest, Hungary, and the honorary doctorate (Doctor Honaris Causa) in 2009.

During his 34-year career in telecommunications, he has held various research and academic posts in Hungary, Germany, and the U.K. Since 1986, he has been with the School of Electronics and Computer Science, University of Southampton, Southampton, U.K., where he holds the chair in telecommunications. He is currently directing an academic research team working on a range of research projects in the field of wireless multimedia communications sponsored by industry, the Engineering and Physical Sciences Research Council U.K., the European First Programme, and the Mobile Virtual Centre of Excellence, U.K. $\mathrm{He}$ is an enthusiastic supporter of industrial and academic liaison, and he offers a range of industrial courses. He has co-authored 20 John Wiley-IEEE Press books on mobile radio communications, totaling in excess of 10000 pages, and has published about 970 research entries on IEEE Xplore.

Dr. Hanzo is a Governor of both the IEEE Communications and the Vehicular Technology Societies. He is the Editor-in-Chief for the IEEE Press and a Chaired Professor also at Tsinghua University, Beijing, China. He has acted as Technical Program Committee Chair of IEEE conferences, presented keynote lectures, and has been awarded a number of distinctions. 